\title{
Genetic and functional analysis of the bovine uterine microbiota. Part II: Purulent vaginal discharge versus healthy cows
}

\author{
M. L. S. Bicalho, S. Lima, C. H. Higgins, V. S. Machado, F. S. Lima, and R. C. Bicalho ${ }^{1}$ \\ Department of Population Medicine and Diagnostic Sciences, College of Veterinary Medicine, Cornell University, Ithaca, NY 14853
}

\begin{abstract}
The aim of this study was to characterize, using metagenomic shotgun DNA sequencing, the intrauterine microbial population and its predicted functional diversity within healthy cows and cows presenting purulent vaginal discharge (PVD). Twenty Holstein dairy cows from a single farm were enrolled in the study at 25 to 35 d postpartum. Purulent vaginal discharge was diagnosed by retrieving and scoring vaginal discharge using the Metricheck device (Simcro, Hamilton, New Zealand). Intrauterine samples for metagenomic analysis were collected by the cytobrush technique from 8 cows diagnosed with PVD and 12 healthy cows. Pair-end sequencing was performed using the Illumina MiSeq platform (Illumina Inc., San Diego, $\mathrm{CA}$ ). Metagenomic sequences were analyzed using the MG-RAST server (metagenomic rapid annotations using subsystems technology; http://metagenomics.anl. gov/), and the STAMP software (http://kiwi.cs.dal. ca/Software/STAMP) was used to study statistically significant differential abundance of taxonomic and functional features between the 2 metagenomes. Additionally, the total number of bacterial $16 \mathrm{~S}$ rDNA copies was estimated by real-time PCR. Taxonomic analysis revealed that Bacteroidetes was the most abundant phylum in the uterine microbiota from cows with PVD, and Fusobacteria was almost completely absent in the healthy uterine microbiota. Moreover, species belonging to the genus Trueperella were present only in the uterine microbiota of PVD cows. The increased abundance of Fusobacteria and the unique presence of Trueperella in the PVD cows highlight the important role of these bacteria in the pathogenesis of PVD. Genes encoding cytolethal distending toxin were exclusive to the microbiota of PVD cows. Similarly, genes associated with lipid A modification were present only in samples from PVD cows; such modification is associated with greater resistance to cationic antimicro-
\end{abstract}

Received September 27, 2016.

Accepted January 14, 2017.

${ }^{1}$ Corresponding author: rcb28@cornell.edu bial peptides. Conversely, genes encoding bacteriocins and ribosomally antibacterial peptide were exclusively found in the healthy uterine microbiota and dominated by tolerance to colicin E2. No difference was observed in total bacterial load between the 2 microbiotas. This study provides deep insight into the uterine microbial community in health and disease. The observations that the healthy microbiota is tolerant to colicin E2, whereas the uterine microbiota of PVD cows produces cytolethal distending toxins and modifies its lipopolysaccharides suggest that species-intrinsic factors may be more relevant than bacterial abundance to the development of disease or maintenance of health in the dairy cow postpartum uterus.

Key words: purulent vaginal discharge, shotgun, microbiome

\section{INTRODUCTION}

Purulent vaginal discharge (PVD) affects 15 to $42 \%$ of dairy cows, generally after the third week of lactation (Potter et al., 2010; Westermann et al., 2010), and it is characterized by presence of purulent or mucopurulent exudates as a result of bacterial infection (Sheldon et al., 2006). Bacteria in the uterus can cause inflammation, histological lesions of the endometrium, and delay in uterine involution (Bonnett et al., 1991; Sheldon et al., 2006). It has been shown that inflammation of the endometrium has a detrimental effect on reproductive performance, reducing both first-service conception rate and the overall pregnancy risk (Kasimanickam et al., 2004; Gilbert et al., 2005).

Several etiological agents have been associated with PVD and endometritis; of these, Escherichia coli, Trueperella pyogenes, and Fusobacterium necrophorum are considered the most relevant pathogenic species in dairy cows because their coordinated action results in inflammation and destruction of the endometrium (Bicalho et al., 2010, 2012; Potter et al., 2010; Santos and Bicalho, 2012; Amos et al., 2014). Conversely, it was found that presence of CNS and $\alpha$-hemolytic streptococci reduces the risk of endometrial inflammation (Sens and Heuwieser, 2013), suggesting that the taxonomic structure of the microbiota influences disease outcome. 
Comprehensive knowledge of the uterine microbiota may provide deeper insights into the ecology of species contributing to health or disease, and potentially into the design of more effective therapeutic interventions (LeBlanc et al., 2006). With advances in technologies such as sequence- and function-based gene screening and high-throughput sequencing, important insights into microbiomes have emerged, including identification of microorganisms associated with uterine health and disease. The diversity and complexity of the microbial denizens of the postpartum uterine environment of dairy cows have been described in studies using metagenomic analysis of the $16 \mathrm{~S}$ rRNA gene (Santos et al., 2011; Machado et al., 2012; Santos and Bicalho, 2012; Peng et al., 2013). However, knowledge of the functional content of the bovine postpartum intrauterine bacterial community is still rudimentary. Wholegenome shotgun sequencing can yield information on gene abundance (e.g., genes associated with virulence factors, metabolism, and antibiotic resistance), thus enabling assessment of biological functions in microbial communities. Additionally, determination of bacterial numbers by real-time quantitative PCR (qPCR) based on the $16 \mathrm{~S}$ rRNA gene is expected to have far greater sensitivity and precision than measurements of microbial relative abundance within the uterine environment (Nadkarni et al., 2002).

Therefore, the primary objective of this study was to characterize the intrauterine microbial population and its predicted functional diversity from healthy cows and cows affected with PVD. We also sought to identify specific microbial genes with a role in postpartum uterine health. An additional feature of this study is that we estimated the total bacterial load in the endometrial samples by using real-time qPCR.

\section{MATERIALS AND METHODS}

\section{Ethics Statement}

This study was carried out in strict accordance with the recommendations of The Animal Welfare Act of 1966 (AWA) (P.L. 89-544) and its amendments, 1970 (P.L. 91-579), 1976 (P.L. 94-279), and 1985 (P.L. 99198), that regulate the transportation, purchase, care, and treatment of animals used in research. The research protocol was reviewed and approved by the Institutional Animal Care and Use Committee of Cornell University (protocol number 2011-0111). Sampling procedures and experimental manipulations were authorized by the farm owner, who was aware of the procedure. No specific permissions were required for these locations or activities. We confirm that the field of the study did not involve endangered or protected species.

\section{Farm Management}

In total, 20 Holstein dairy cows at 25 to 35 d postpartum from a single farm located near Ithaca, New York, were enrolled from June 7 to 14, 2014. The farm milks 3,450 Holstein cows 3 times daily in a parallel rotary 100-stall milking parlor, and milk production is automatically recorded on a daily basis. Primiparous and multiparous cows are housed in freestall barns, with concrete stalls covered with mattresses and bedded with manure solids. Cow reproductive management was based on a combination of Presynch (Moreira et al., 2001), Ovsynch (Pursley et al., 1995), Resynch (Fricke et al., 2003), and detection of estrus.

\section{Case Definition}

At 25 to 35 d postpartum, PVD diagnosis was performed by one investigator, who assessed the presence of a purulent or mucopurulent secretion in the vaginal discharge sample using a Metricheck device (SimcroTech, Hamilton, New Zealand). Vaginal discharge was scored using a modified 0 to 5 scale: $0=$ no secretion material retrieved, $1=$ clear mucus, $2=$ flecks of pus in the vaginal discharge, $3=<50 \%$ pus in the vaginal discharge, $4=>50 \%$ pus in the vaginal discharge, $5=$ watery, fetid vaginal discharge (McDougall et al., 2007; Dubuc et al., 2010). Cows with a score $\geq 3$ were classified as PVD cows $(n=12)$; cows with a score $\leq 2$ were classified as healthy $(\mathrm{n}=8)$.

\section{Sample Collection}

For metagenomics sequencing, intrauterine samples were obtained using the previously described cytobrush technique (Kasimanickam et al., 2004). Briefly, cows were restrained and the perineum area was cleansed and disinfected with a $70 \%$ ethanol solution. In the cow, uterine lavage and cytobrush cytology have been accepted as less invasive methods of endometrial sampling. Although the lavage technique allows an increase in the area of endometrium sampled, we anticipated that collecting a sample of mucus and exudates from a large uterine surface would hamper DNA extraction and purification procedures. Although the cytobrush technique requires specialized equipment, this was the method of choice for use on the field, because it is considered easier, more consistent, and faster than the lavage method. A modified gynecologic sterile brush protected by a disposable plastic catheter and a plastic sleeve was introduced into the uterine body through the vagina. At the external os of the cervix, the outer plastic sleeve was perforated and the disposable sheath and extension were passed through the cervix. Upon 
reaching the innermost ring of the cervix, the cytobrush was advanced approximately $1 \mathrm{~cm}$ beyond the sheath into the lumen of the uterine body and rolled onto the uterine wall. The brush was retracted into the catheter and the device was removed from the reproductive tract. Immediately after sampling, the cytobrush was transferred to a DNase-/RNase-free 2-mL microcentrifuge tube and immersed in $1.5 \mathrm{~mL}$ of DNase-/RNase-/ DNA-/RNA-free water. Samples were kept on ice until they were transferred to a $-80^{\circ} \mathrm{C}$ freezer.

\section{DNA Extraction and Whole-Genome Shotgun Sequencing}

Before DNA extraction, the uterine samples were thawed, vortexed, and settled in a Mini-Beadbeader-8 (Biospec Products, Bartlesville, OK) to disperse mucus, bacteria, and cells. The brushes were removed from the tubes and selective lysis of eukaryotic cells was performed by osmotic shock as applied in other studies (Hunter et al., 2011). Bacterial cells were then collected by centrifugation the suspension was centrifuged at $13,000 \times g$ for $10 \mathrm{~min}$, and total DNA was extracted from the pellet. Then, DNA extraction was performed using a PowerSoil DNA Isolation Kit (MO BIO Laboratories Inc., Carlsbad, CA), following the manufacturer's recommendations. The concentration of DNA was evaluated using a Quant-iT PicoGreen dsDNA Assay Kit (Life Technologies Corp., Carlsbad, CA).

Additionally, the phylogenetic characterization of the metagenome community was confirmed by amplification of the V4 hypervariable region of the bacterial 16S rDNA genes amplified by PCR according to a previously described protocol and optimized for the Illumina MiSeq platform (Illumina Inc., San Diego, CA) using different 12-bp error-correcting Golay barcodes for the 16S rRNA gene PCR (Caporaso et al., 2012). Details on library preparation, assessment of the resulting metagenome sequence data, and bioinformatics are similarly described in the companion paper (Bicalho et al., 2017). Briefly, through MG-RAST analysis, sequences were subjected to quality control, which included dereplication (removal of erroneous sequences produced by sequencing artifacts), removal of hostspecific sequences (Bos taurus, UMD v3.0; ftp://ftp. cbcb.umd.edu/pub/data/Bos_taurus/latest_version/ README.txt), ambiguous base filtering (removing sequences with $>5$ ambiguous base pairs), and length filtering (length filters discard sequences whose length differs from the average sequence length by more than the specified number of standard deviations). The nonredundant, multi-source protein annotation database (M5NR; Wilke et al., 2012) was the annotation source used for organism classification. Subsystems under SEED were used for functional abundance analysis.

\section{Determination of Bacterial Load by Quantification of 16S rDNA Copy Number in Uterine Samples}

To determine the bacterial load of the uterine samples, we first cloned (into TOP10 cells) a plasmid containing the amplified V6 hypervariable region by using the Zero Blunt TOPO PCR cloning kit (Life Technologies, Darmstadt, Germany). Plasmid was purified with a QIAprep Spin Miniprep Kit (Qiagen, Valencia, $\mathrm{CA}$ ) and quantified using Quant-iT PicoGreen and a dsDNA Broad Range Assay Kit (Life Technologies Corporation). Insertion was confirmed by agarose gel electrophoresis, and by pyrosequencing at the Cornell University Life Science Core Laboratories Center.

The 16S RNA gene copy numbers were measured by qPCR using Unibac primers (forward: 5'-TGGAGCATGTGGTTTAATTCGA-3'; reverse: 5'-TGCGGGACTTAACCCAACA-3'). The PCR were performed in 15$\mu \mathrm{L}$ volumes consisting of $1 \times$ iQTMSybr Green Supermix (Bio-Rad Laboratories, Hercules, CA), $300 \mathrm{nmol}$ of each primer and $50 \mathrm{ng}$ to $5 \mathrm{pg}$ of the genomic DNA (or plasmid DNA standards). The thermal cycler conditions were as follows: denaturation at $95^{\circ} \mathrm{C}$ for $3 \mathrm{~min}$, 40 amplification cycles $\left(95^{\circ} \mathrm{C}\right.$ for $10 \mathrm{~s}, 55^{\circ} \mathrm{C}$ for $\left.30 \mathrm{~s}\right)$, and 2 final steps at $95^{\circ} \mathrm{C}$ for $1 \mathrm{~min}$ and $55^{\circ} \mathrm{C}$ for $1 \mathrm{~min}$ followed by a melt curve. All reactions were performed in duplicate using a MyiQTM Real-Time PCR Detection System (Bio-Rad Laboratories). Quantification of 16S RNA gene target DNA was achieved by 10-fold serial dilutions ranging from $10^{0}$ to $10^{7}$ plasmid copies of the previously quantified plasmid standards. Plasmid standards and uterine samples were run in duplicate. The average of the cycle threshold value was used for calculation of the bacterial load.

\section{Statistical Analysis}

Sequencing reads from the DNA libraries were used to determine the phylogenetic composition and functional distribution of the uterine microbiomes of the respective health status groups. The STAMP software v2.0 (http://kiwi.cs.dal.ca/Software/STAMP) was used to study statistically significant differential abundance of taxonomic and functional features between the 2 metagenomes (Parks et al., 2014). Statistical analyses involving Welch's test with Benjamin-Hochberg false discovery rate correction were applied at level 1 of functional annotations to derive statistically significant differential features between the 2 uterine metagenomes (PVD and healthy cows). The results were filtered using a $P$-value of 0.05 and an effective size of 0.5 thresh- 
old in STAMP. An analysis of similarity (ANOSIM) multivariable test was used to test for similarities at level 2 of functional annotations between the 2 metagenomes. The $P$-values were used to denote the statistical significance of the ANOSIM R statistic values found when comparison between differential features from PVD and healthy metagenomes were performed. The $R$ values indicate the degree of functional genes separation across health status; values approaching unity $(\mathrm{R}=1)$ indicate clear separation, whereas $\mathrm{R}=0.5$ indicates dissimilarity with some overlap.

Quantitative real-time PCR was used to monitor the amplification of the $16 \mathrm{~S}$ rRNA gene during PCR. This method yields an absolute quantification of the number of target DNA molecules within a sample based on comparison with DNA standards (serial dilution of our 16S rDNA gene clone) using a calibration curve. The total bacterial load of each endometrial sample was defined as the $\log _{10}$ copy number of the $16 \mathrm{~S}$ rDNA gene. Student's $t$-test was used to compare differences in total bacterial load between uterine samples from cows diagnosed with PVD and healthy cows.

\section{RESULTS}

\section{Sequencing Results and Phylogenetic Distribution in the Uterine Microbiome}

Metagenome sequencing of endometrial samples from the 12 PVD and 8 healthy cows yielded a total of 9,602,142 raw reads and approximately 7 million reads after quality filtering. The sequencing data of the individual samples are detailed in Supplemental Table S1 (https://doi.org/10.3168/jds.2016-12061). Based on the MG-RAST annotation, about 0.1 to $0.5 \%$ of the reads were classified as rRNA per uterine microbiome sample, and 75 to $95 \%$ of the reads from all samples were classified into various functional subsystems. We obtained a combined total of approximately 1,756,282 high-quality bacterial reads that were classified into various functional subsystems and 20,334 high-quality $16 \mathrm{~S}$ rDNA sequence reads. The rRNA-similar reads were then clustered at $97 \%$ identity, and the longest sequence was picked as the cluster representative.

A BLAST-like alignment tool (BLAT; Kent, 2002) similarity search for the longest cluster representative was performed against the M5rna database which integrates Silva (Pruesse et al., 2007), Greengenes (DeSantis et al., 2006), and RDP databases (Cole et al., 2009). More than $80 \%$ of uterine sample reads were annotated to the domain level according to the M5NR database (Wilke et al., 2012). To assess the depth of sequencing performed for each sample, comparative rarefaction curves were generated using MG-RAST. Rarefaction analysis showed that, for all samples, the number of sequences screened was sufficient to determine the total number of sequence types within these samples, as they reached a plateau (data not shown). Bacteria and Eukaryota were the most abundant domains, followed by the Viruses and Archaea. The most abundant phylum in the Eukaryota domain was Chordata, which is most likely bovine DNA. Samples were then ranked based on the mean relative abundance at the phylum and genus levels.

\section{Bacterial Phylogenetic Profile}

Sequences from uterine samples of PVD cows predominantly contained phylotypes affiliated with Bacteroidetes, Proteobacteria, Firmicutes, Actinobacteria, Fusobacteria, and Spirochaetes (Figure 1). Twenty-one phyla each with a prevalence $<3.0 \%$ were combined and designated as "others." In healthy cows the same phyla were present; however, the distribution of these phyla was different. Fusobacteria were almost absent in the healthy bovine uterine microbiota, representing less than $1.0 \%$ of the bacterial population. Concomitantly, there was a substantial and statistically significant increase $(P \leq 0.05)$ in Fusobacteria in PVD cows uterine microbiota cows, representing $6 \%$ of the total bacteria population.

The proportion of Bacteroidetes in healthy cows was $20 \%$, whereas it was $29 \%$ in PVD cows $(P=0.27)$. Conversely, the relative abundance of sequences affiliated with Proteobacteria was $33 \%$ in healthy cows, but fell to $28 \%$ in PVD cows $(P=0.63)$.

The most abundant bacterial taxa identified in the uterine microbiota of healthy and PVD cows are depicted in Figure 2A, which illustrates differential representation by mean proportion and their differences between the 2 health statuses. Bacteroides, Fusobacterium, Bacillus, and Leptotrichia were the most abundant genera in uteri of PVD cows $(P=0.199, P=0.1, P=$ 0.7 , and $P=0.17$, respectively). The most abundant bacterial genera in healthy uteri include Escherichia, Shigella, and Pseudomonas $(P=0.18 ; P=0.18$; and $P$ $=0.28$, respectively).

The distribution of selected genera is depicted in Figure 2B. Trueperella species were absent from the healthy uterine microbiota $(P \leq 0.16)$.

\section{Archaeal and Viral Phylogenetic Profiles}

Archaea accounted for a low percentage of the total community $(0.01 \%)$. The most common archaeal species in the uteri of PVD and healthy cows were Methanobrevibacter smithii and Methanobrevibacter ruminantium. Bovine herpesvirus type 4 ranked among 


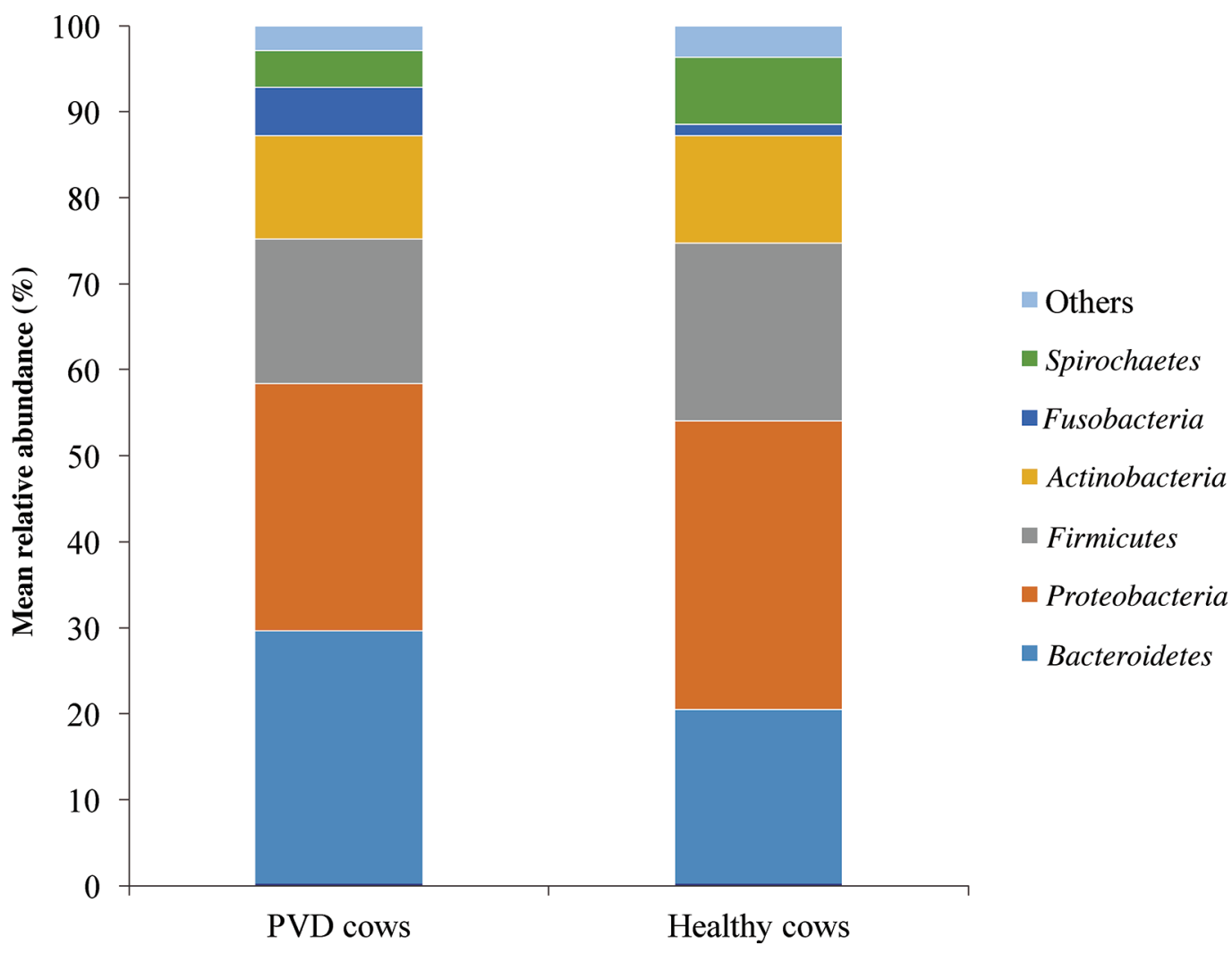

Figure 1. Histograms representing the overall relative distribution of bacteria at the phylum level in uterine samples from cows with purulent vaginal discharge (PVD) and healthy cows at 25 to $35 \mathrm{~d}$ postpartum. Shading was assigned to phyla that constituted $\geq 1.0 \%$ of all sequences observed within each health status. Color version available online.

the 25 most abundant viruses in both microbiota. Bovine herpesvirus type 4 and leukemia virus had numerically higher mean relative abundances in PVD uterine samples compared with healthy samples; however, the differences were not significant. Because of their low prevalence (based on mean relative abundance and number of sequence reads), both the Archaea and Virus domains were excluded from all disease association analyses. The presence of such microbes in the uterine environment was not sufficiently robust to be able to discern whether they favored specific types of bacteria; however, the possibility of syntrophic relationships among these microbes in the reproductive tract may be worth investigating.

\section{Functional Profile of the Uterine Microbial Community at 25-35 d Postpartum}

The SEED database arranges metabolic pathways into a hierarchical structure in which all of the genes required for a specific task are categorized into subsystems and ranked by levels from 1 to 3 (Overbeek et al., 2014). Functional analysis at level 1 using the SEED database in MG-RAST revealed that, among the func- tional systems that were identified in bovine uteri, the most predominant, as expected, were basic functional genes such as those involved in metabolism of carbohydrates, proteins, and amino acids and derivatives. Also identified were genes assigned to clustering-based systems (i.e., systems that are independently grouped with no previous specific classification). These level 1 reads accounted for 12 to $21 \%$ of the total reads referred to the functional profile.

The healthy uterine microbiota demonstrated a numerically higher mean relative abundance compared with PVD cows for most of the functional systems identified; however, this difference was not significant (Figure 3A, $P=0.4$ ). Further analysis was performed to better understand the predicted functional differences between the uterine microbiota of healthy and PVD cows that may generate a hypothesis regarding the role of the different community members in controlling the fate of infection. We completed phylogenetic analysis of the uterine community composition at levels 2 and 3 of specific functions that we believe may represent molecular mechanisms of microbial remodeling used by bacteria to invade host tissues and evade the immune system. Figure 3B depicts extended error bar plots of 
A)

PVD $\square \mathrm{H}$

Bacteroides

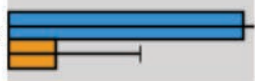

Escherichia

igella

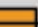

Fusobacterium $\square$

Pseudomonas

Acinetobacter

Leptotrichia

Streptobacillus

Treponema 昌

Staphylococcus th

Salmonella

Bacillus

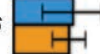

Prevotella

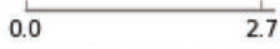

Mean proportion (\%)

B)

PVD $\square \mathrm{H}$

Actinomyces

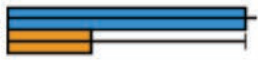

Arcanobacterium

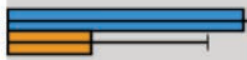

Trueperella

Mobiluncus
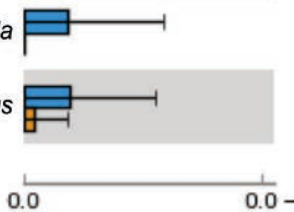

Mean proportion (\%)
$95 \%$ confidence intervals

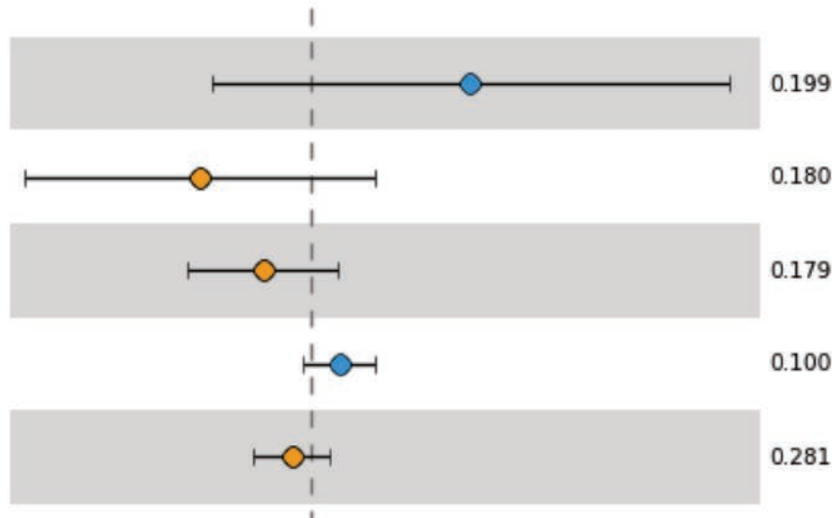

0.303 จิ

0.171 웅

0.171 年

0.475

0.234

0.200

0.731

0.772

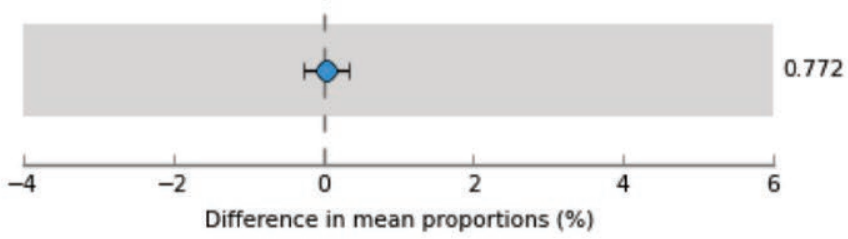

$95 \%$ confidence intervals

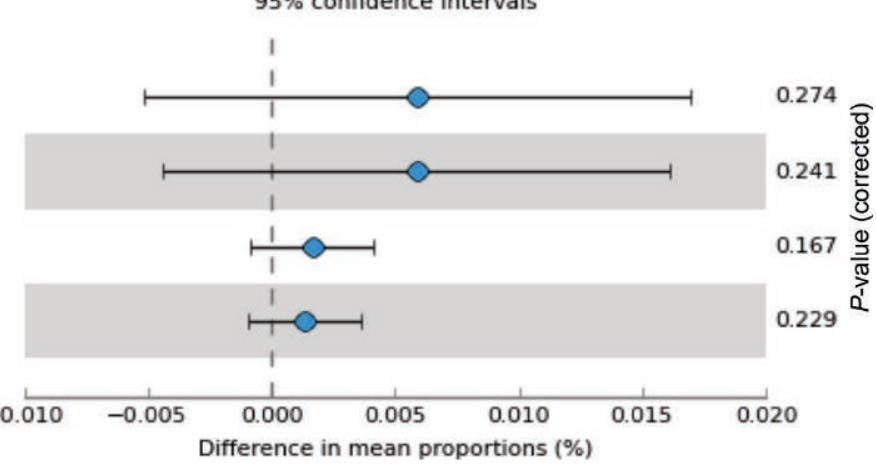

Figure 2. Extended error bar plots illustrating the mean proportion and their differences in (A) bacterial genera most common in uterine samples from cows with purulent vaginal discharge (PVD) and healthy (H) cows at 25 to 35 d postpartum, and (B) genus distribution within the Actinobacteria phylum. Taxa were ranked by respective $P$-values and effect size of 0.5 thresholds in STAMP (Statistical Analysis of Metagenomic Profiles; http://kiwi.cs.dal.ca/Software/STAMP). Circles represent the difference between the mean proportion of bacteria (the effect size), adjacent to their respective CI (error bars). Subsystems overrepresented in the PVD and healthy microbiota have a positive or negative difference between proportions and are indicated by blue (dark) and orange (white) coloring, respectively. Color version available online. 
abundance profiles at level 1 of the uterine community composition for selected predicted functional genes. Based on those findings, we undertook further analysis at level 2 of the specific functional gene categories (a) cell wall and capsule, (b) virulence, disease and defense, (c) DNA metabolism, and (d) stress response, and compared the results between each health status samples within each functional category (Figure 4A-D).

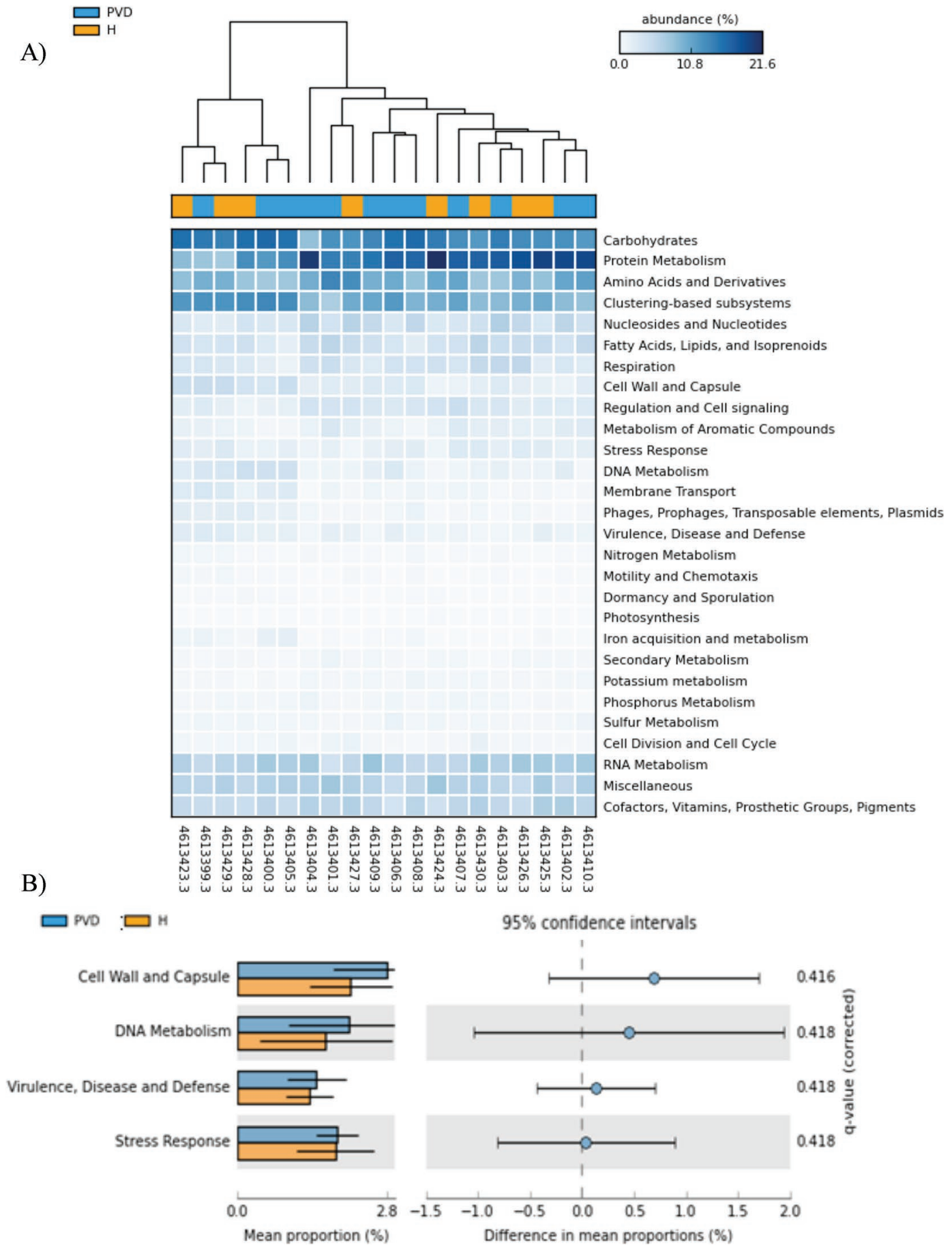

Figure 3. Distribution of functional annotations of uterine metagenomic data from cows with purulent vaginal discharge (PVD) and healthy (H) cows. The data were normalized in the MG-RAST (metagenomic rapid annotations using subsystems technology) pipeline and represent the differential distribution of functional categories. (A) Heatmap dendrogram showing functional category assignments at subsystem level 1 ranked by relative abundance; (B) extended error bar plots depicting the abundance profile of the predicted functional genes. Q-values are adjusted $P$-values using an optimized false discovery rate approach. Color version available online. 


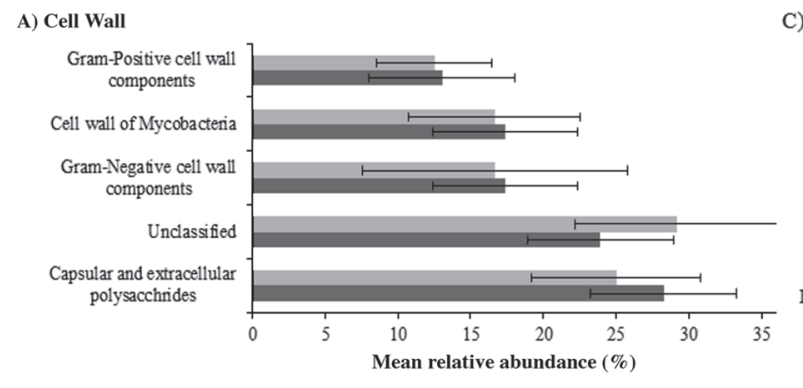

B) DNA metabolism
C) Virulence

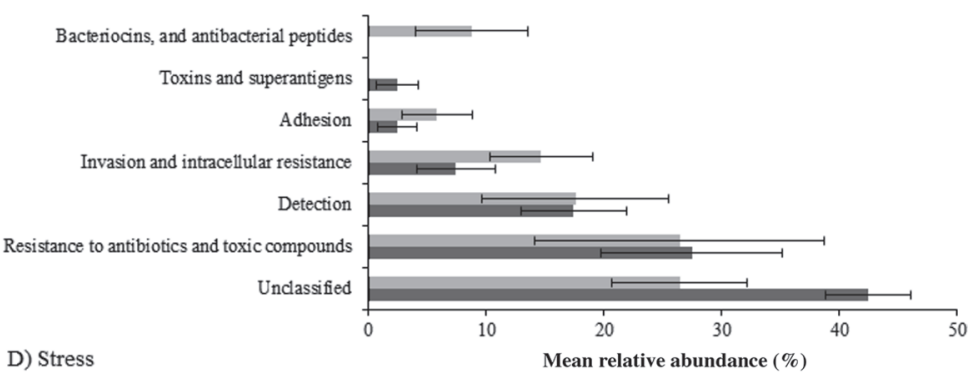

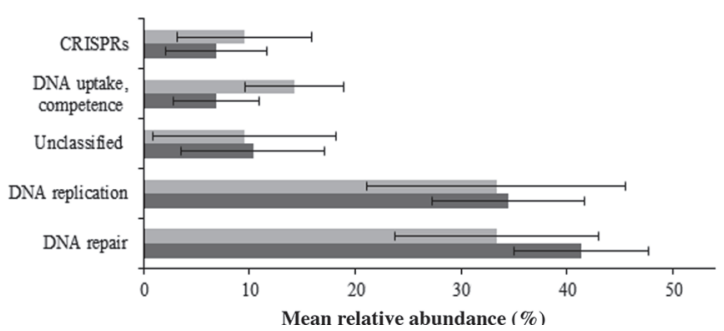

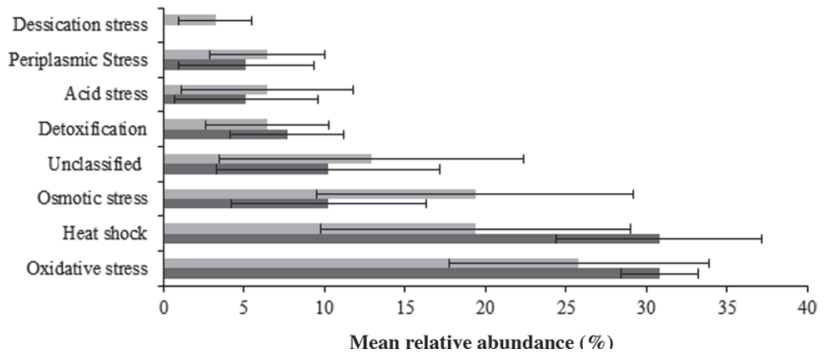

Figure 4. Subsystems of selected functional categories identified in the uterine microbiota of cows with purulent vaginal discharge (dark gray bars) and healthy (light gray bars) cows. The x-axis represents mean relative abundance and the error bars are SEM.

Figure 4A illustrates prokaryotic structural components present in both metagenomes. Capsular and extracellular polysaccharide genes (genes associated with cell wall structural variability besides LPS and lipoteichoic acid were numerically higher in the microbiota of PVD cows, and were mostly characterized by sialic acid biosynthesis (ANOSIM $P=0.2, \mathrm{R}=0.6$ ). Gram-negative cell wall components (LPS) were found to be slightly higher in the microbiota of PVD cows than in the healthy microbiota and were dominated by LPS assembly and lipid A biosynthesis. Interestingly, genes encoding lipid A modification were present only in samples of PVD cows (ANOSIM $P=0.17, \mathrm{R}=0.8$ ). The DNA metabolism system was composed of DNA repair, DNA replication, DNA uptake and competence, DNA recombination and clustered regularly interspaced short palindromic repeats (CRISPR; Figure 4B). The healthy microbiota presented a higher average relative abundance for all DNA categories compared with the microbiota of PVD cows; however, the difference within each of those categories was not significant $(P=0.54)$.

"Resistance to antibiotic and toxic compounds" was the predominant subsystem identified in the virulence, disease, and defense systems category $(>50 \%)$ for the uterine microbiota of both the PVD cows and healthy cows (Figure 4C). The toxins and superantigens function was found only in the uterine microbiota of the PVD cows (ANOSIM $P \leq 0.05, \mathrm{R}=0.86$ ). Cytolethal distending toxins (CDT) were predominant within this category. Bacteriocins and ribosomally synthesized antibacterial peptides were exclusive to the healthy uterine microbiota and were dominated by tolerance to colicin E2 (ANOSIM $P \leq 0.05, \mathrm{R}=0.78$ ). The bacterial adaptive response to oxidative stress and heat shock functions were numerically higher in the uterine microbiota of PVD cows than in the healthy microbiota (ANOSIM $P=0.28, \mathrm{R}=0.4$; Figure $4 \mathrm{D}$ ).

Relative abundance of sequences encoding for lipid A modification and CDT representing 0.001 and $0.015 \%$, respectively, of the total number of sequences affiliated to functional profile enclosed by PVD cows are depicted in Figure 5.

\section{Total Bacterial 16S rDNA Load}

To compare the total bacterial load between uterine samples from cows with PVD and healthy cows, we assessed the number of $16 \mathrm{~S}$ rDNA copies (V3 region) per milliliter of uterine sample. Values for copies/milliliter of sample were log-transformed before analysis to pass the normality test. Differences between mean values of the 2 groups were analyzed with a Student's $t$-test. No statistical difference was observed between uterine microbiota of PVD and healthy cows $(P=0.87)$. Results are shown as the mean values of 2 replicates \pm SEM (Figure 6).

\section{DISCUSSION}

In this study, we conducted a sequence-and-functionbased gene evaluation of the uterine microbiome and its association with late infection of the uterus of 


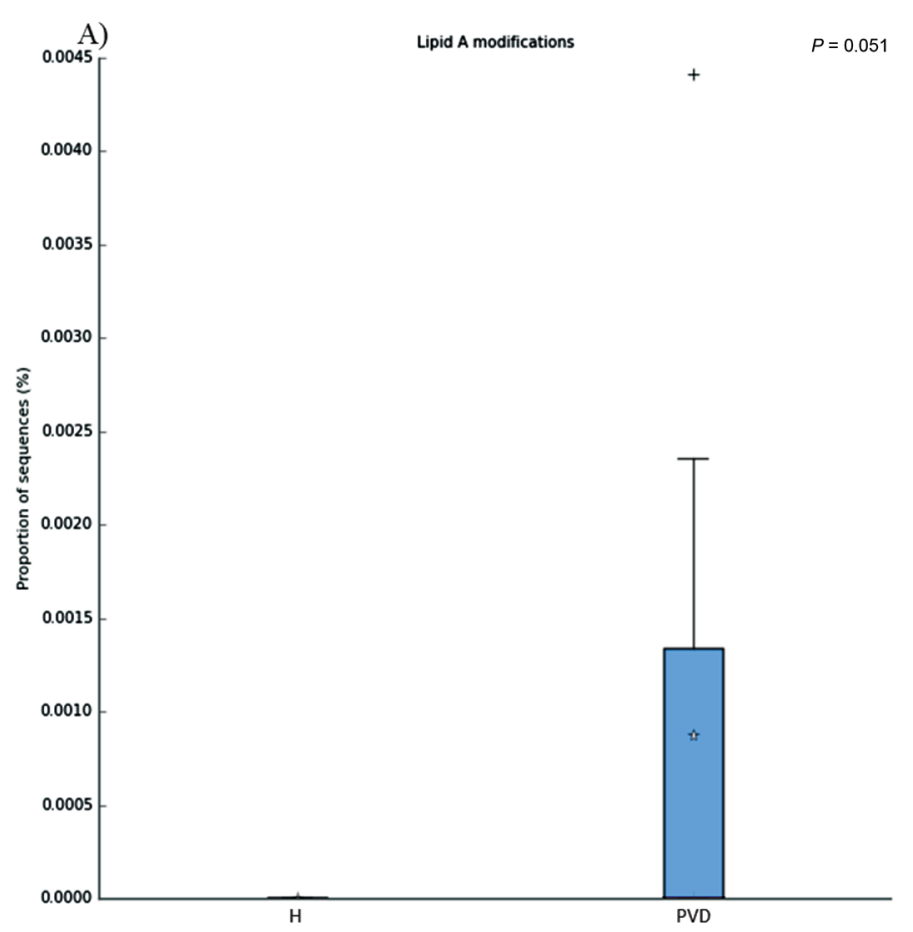

B)

Cytolethal distending toxins

$P=0.423$

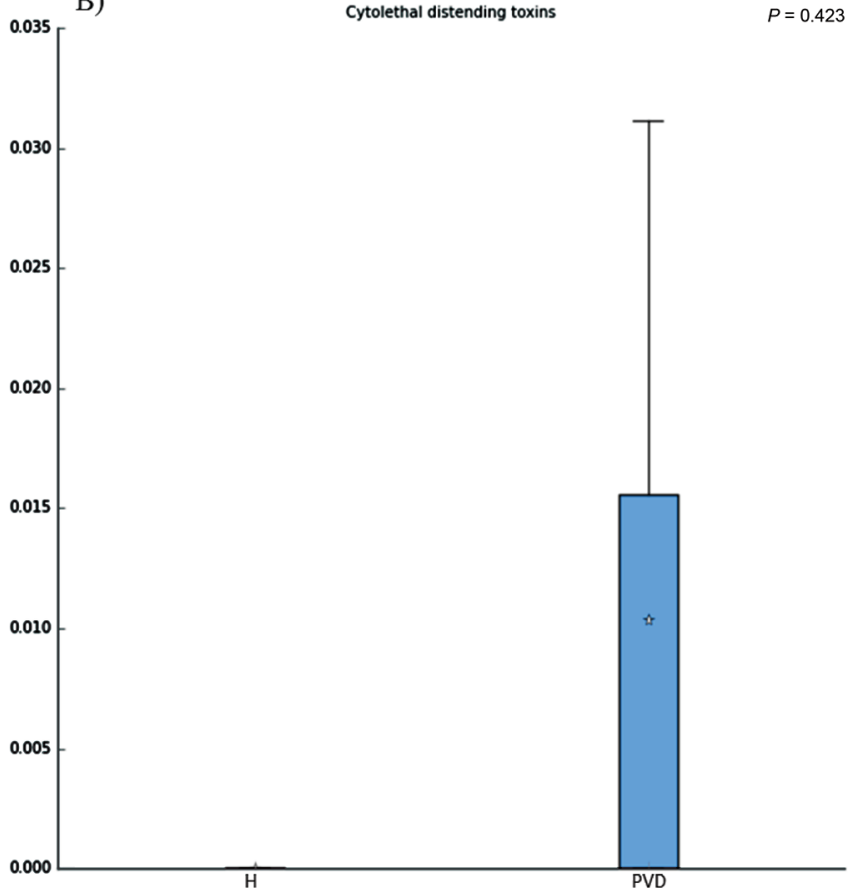

Figure 5. Box plot showing the distribution of the functional categories at subsystem level 2 of uterine metagenomic data for purulent vaginal discharge (PVD) cows and healthy $(\mathrm{H})$ cows. The data were normalized in the MG-RAST (metagenomic rapid annotations using subsystems technology) pipeline and ranked by relative abundance using STAMP software (Statistical Analysis of Metagenomic Profiles; http://kiwi.cs.dal. $\mathrm{ca} /$ Software/STAMP). The unique presence and relative abundance of genes encoding for tolerance to lipid A modification and cytolethal distending toxins (PVD microbiota) are represented in panels A and B, respectively. Boxes indicate the interquartile range (IQR; 75th to 25th of the data). The mean value is shown as a star. Whiskers extend to the most extreme value within $1.5 \times \mathrm{IQR}$, and outliers are shown as crosses. Color version available online.

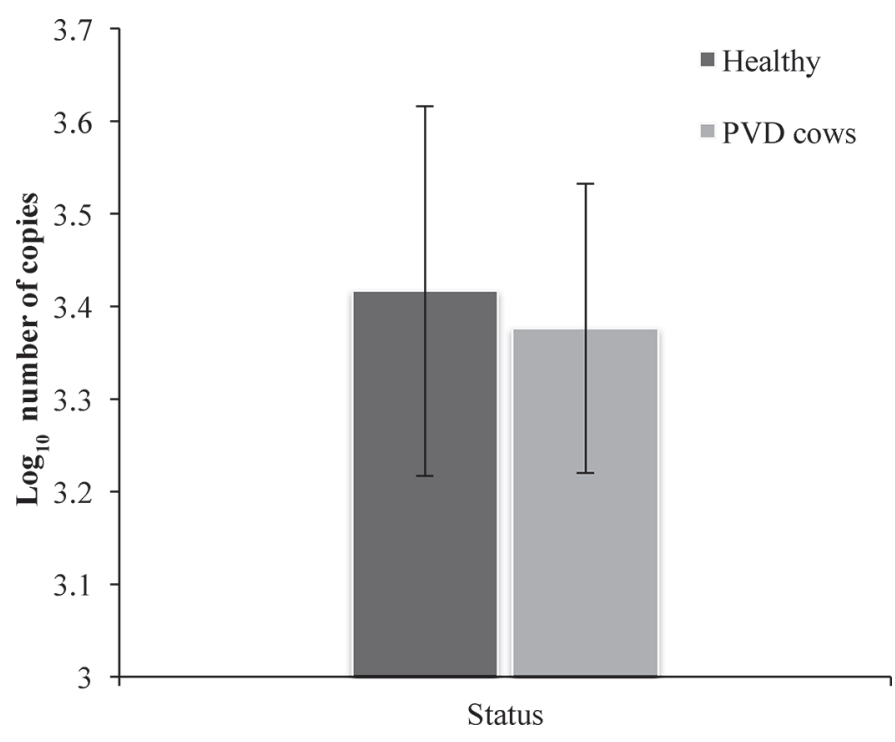

Figure 6. Number of bacterial $16 \mathrm{~S}$ ribosomal DNA copies (V6 region) in uterine samples from cows with purulent vaginal discharge (PVD) and healthy cows. Values for copies per milliliter of sample were log-transformed before analysis to pass the normality test. Differences between mean values of the 2 groups were analyzed with Student's $t$ test. No statistical difference was observed between PVD and healthy uterine microbiota $(P=0.87)$. Error bars are SEM.
Holstein cows. Our data demonstrating the increased abundance of Bacteroidetes and Fusobacteria coupled with the unique presence of Trueperella in the uterine microbiota found in cows presenting PVD highlight the important role of those bacteria in the pathogenesis of PVD. Nevertheless, in the present study, total bacterial load in the uterus was not associated with presence of pus in the vagina of dairy cows. Additionally, when we assessed for the presence of genes coding for molecular mechanisms that may enhance microbial pathogenicity, genes for LPS modification and CDT were exclusive to the uterine microbiota of PVD cows. Our findings indicate that species specificity and species intrinsic factors may be more relevant than bacterial load to the development and appearance of pus in the cow postpartum vaginal discharge.

The above results are in line with those of Santos and Bicalho (2012), who reported evidence for a defined bacterial core assemblage in the uterus, markedly evident at $35 \mathrm{~d}$ postpartum, of which Bacteroidetes and Fusobacteria dominate in cows diagnosed with endometritis and pyometra (Santos and Bicalho, 2012; Knudsen et al., 2015). In addition to the presence of $T$. pyogenes, abundance of Bacteroidetes and Fusobacteria 
in the uterus has been a common finding in cows with PVD, and is frequently associated with uterine inflammation (Williams et al., 2005; Wagener et al., 2014; Machado et al., 2012; Bicalho et al., 2016).

It is noteworthy that, in the present study, Trueperella was not among the most common genera found in either uterine microbiota (healthy and PVD cows), constituting only $1 \%$ of the total number of sequences, which is similar to the results of Knudsen et al. (2015). Indeed, the low prevalence of Trueperella, even in PVD cows or endometritic samples, is not a new observation. For example, when Machado et al. (2012) investigated the bacterial diversity of the postpartum uterus of dairy cows by using metagenomic pyrosequencing, the presence of the genus Arcanobacterium (now Trueperella) was associated with endometritis, even with a relative abundance of only 2\% in PVD uteri. However, also noteworthy in the current study was the absence of Trueperella species in healthy cows, which further supports the important etiologic role of this genus in the pathogenesis of PVD.

Pathogenic strains differ from harmless commensals by containing more genomic DNA encoding virulence factors such as fimbrial adhesins, capsules, iron sequestration systems, toxins, mammalian cell invasion factors, and zones of recombination (Welch et al., 2002). Capsular polysaccharides are among the bestunderstood virulence factors in terms of resistance to innate immunity (Deszo et al., 2005). In the present study, genes encoding capsular- and extracellular polysaccharide-related functions were found mainly in the uterine microbiota of PVD cows, and sialic acid biosynthesis was the most prevalent. Sialic acid is produced by certain pathogenic microorganisms and is considered to play an essential role in disease pathogenesis by mimicking the invading bacteria and helping them to elude host immune mechanisms (Vimr and Lichtensteiger, 2002).

Another bacterial strategy that promotes survival in the host is lipid A remodeling. Modification systems are variable and often regulated by environmental conditions (Raetz et al., 2007). Lipid A is a potent antigenic component of LPS in gram-negative bacteria, inducing macrophages to synthesize potent mediators of inflammation (e.g., TNF- $\alpha$ and IL-1; Beutler and Cerami, 1988). As such, lipid A-related functions could be ideal targets for the development of new antibiotics (Raetz et al., 2007). In the present study, lipid A modification functions were uniquely found within the uterine microbiota of PVD cows, and included genes coding for enzymes that modify lipid A with phosphoethanolamine. This latter structural change has been shown to reduce bacterial susceptibility to cationic an- timicrobial peptides and polymyxin, and to contribute to increased pro-inflammatory signaling (Helander et al., 1995; Nummila et al., 1995; Gunn et al., 1998). These observations add to our microbiological results suggesting that the uterine microbiota of PVD cows is characterized by a core of specific microbial activities that promote bacterial escape from host defenses and certain antimicrobial drugs, as is imperative in the pathogenesis of persistent or chronic infection.

In recent years, major advances have occurred in our understating of the pathogenic role of $E$. coli LPS regarding uterine innate immunity of dairy cows; both the severity of disease and the causal relationship between uterine infection and infertility have been linked to the mechanism of LPS pathogenicity (Sheldon and Roberts, 2010; Sheldon et al., 2010; Bromfield et al., 2015). Similar to E. coli, as with other gram-negative bacteria, the outer membrane of $F$. necrophorum contains endotoxic LPS. Moreover, the antigenic potential of $F$. necrophorum LPS to induce necrosis and liver abscesses in cattle has been reviewed (Tadepalli et al., 2009). Although, the role of F. necrophorum LPS in the development of uterine infection needs to be evaluated in detail, severe uterine infections (characterized by fetid purulent vaginal discharge and toxemia) as well as reproductive failure have long been suggested to be dependent on the pathogenic synergism between F. necrophorum and T. pyogenes (Williams et al., 2005; Azawi, 2008; Machado et al., 2012; Jeon et al., 2015).

Several studies have attempted to elucidate the etiological role of $E$. coli and closely related species in the development of uterine infection. For example, presence of the adhesin-encoding gene fim $H$ at least doubled the odds of endometritis (when detected within the first week of parturition) and decreased the likelihood of pregnancy in dairy cows (Bicalho et al., 2010, 2012). Different gram-negative pathogenic bacterial strains are capable of producing CDT that cause characteristic enlargement and death of mammalian cells (De Rycke and Oswald, 2001). The $c d t$ gene is widely present in various $E$. coli pathotypes, mainly in extra-intestinal pathogenic E. coli (ExPEC) strains (Toth et al., 2003), which are known to be associated with endometritis in dairy cattle (Bicalho et al., 2010). So far, no grampositive CDT-producing bacteria have been characterized ( et al., 2014).

Even though in the present study the unique presence of intrauterine $T$. pyogenes highlights the importance of this pathogen in the establishment of PVD, it is important to acknowledge that uterine infection is caused by mixed contamination. These types of observations raise questions of whether the microbiota plays an active causative role in defining the status of the disease or 
whether microbial imbalances and overgrowth of opportunistic bacteria are simply a consequence of the disease.

Curiously, presence of the plo gene, which encodes the hemolytic exotoxin pyolysin and has been consistently associated with Trueperella pyogenes pathogenic action (Bicalho et al., 2012; Amos et al., 2014), was not detected within the toxins subsystem in our study. We acknowledge that the lack of detection of plo might be due to a potential bias, such as suppression of lowabundance species, genome structure, pipeline engineering, and the character of the sequence databases that were used for annotation in the present study. In vitro techniques to minimize the amount of host DNA in our samples were used, as well as selected in silico screening against reads matching the bovine genome. However, metagenome analysis of the endometrial microbiome is unavoidably contaminated by host reads in addition to the increased amount of host sample collection afforded by the cytobrush method used in the present study, thus reducing the sequencing coverage, which might have affected our ability to detect specific genes. Further studies are needed to establish more precise sampling criteria for interpretation of endometrial microbiome.

Finally, genes for bacteriocins and ribosomally antibacterial peptide were exclusively found in the healthy uterine microbiota and dominated by tolerance to colicin E2. Colicins are antimicrobial toxins produced by $E$. coli and active against closely related species by causing extensive degradation of DNA and bacterial cell death (Herschman and Helinski, 1967). We propose that bacteriocins and antimicrobial molecules released by certain commensal members of the uterine community might play a role in repressing the proliferation and overgrowth of potential pathogens. The presence specifically of $E$. coli late postpartum and its association with uterine health have been previously discussed elsewhere (Santos et al., 2011; Bicalho et al., 2012; Jeon et al., 2015).

\section{CONCLUSIONS}

Our study provides deeper insight into uterine microbial community in healthy and PVD cows. The increased abundance of Fusobacteria and the unique presence of Trueperella in the uterine microbiota of PVD cows highlight the important role of these bacterial taxa in the pathogenies of purulent vaginal discharges. Moreover, our observations that total bacterial load was not associated with PVD and that the uterine microbiota of PVD cows produces CDT and modifies its LPS suggest that factors intrinsic to species may be more relevant than bacterial abundance to the development of PVD versus maintenance of health in the cow postpartum uterus.

\section{REFERENCES}

Amos, M. R., G. D. Healey, R. J. Goldstone, S. M. Mahan, A. Duvel, H. J. Schuberth, O. Sandra, P. Zieger, I. Dieuzy-Labaye, D. G. Smith, and I. M. Sheldon. 2014. Differential endometrial cell sensitivity to a cholesterol-dependent cytolysin links Trueperella pyogenes to uterine disease in cattle. Biol. Reprod. 90:54.

Azawi, O. I. 2008. Postpartum uterine infection in cattle. Anim. Reprod. Sci. 105:187-208.

Beutler, B., and A. Cerami. 1988. Tumor necrosis, cachexia, shock, and inflammation: A common mediator. Annu. Rev. Biochem. 57:505-518.

Bezine, E., J. Vignard, and G. Mirey. 2014. The cytolethal distending toxin effects on mammalian cells: A DNA damage perspective. Cells 3:592-615.

Bicalho, M. L., V. S. Machado, C. H. Higgins, F. S. Lima, and R. C. Bicalho. 2017. Genetic and functional analysis of the bovine uterine microbiota. Part I: Metritis versus healthy cows. J. Dairy Sci. 100:3850-3862. https://doi.org/10.3168/jds.2016-12058.

Bicalho, M. L., V. S. Machado, G. Oikonomou, R. O. Gilbert, and R. C. Bicalho. 2012. Association between virulence factors of Escherichia coli, Fusobacterium necrophorum, and Arcanobacterium pyogenes and uterine diseases of dairy cows. Vet. Microbiol. 157:125-131.

Bicalho, R. C., V. S. Machado, M. L. Bicalho, R. O. Gilbert, A. G. Teixeira, L. S. Caixeta, and R. V. Pereira. 2010. Molecular and epidemiological characterization of bovine intrauterine Escherichia coli. J. Dairy Sci. 93:5818-5830.

Bonnett, B. N., S. W. Martin, V. P. Gannon, R. B. Miller, and W. G. Etherington. 1991. Endometrial biopsy in Holstein-Friesian dairy cows. III. Bacteriological analysis and correlations with histological findings. Can. J. Vet. Res. 55:168-173.

Bromfield, J. J., J. E. Santos, J. Block, R. S. Williams, and I. M. Sheldon. 2015. Physiology and Endocrinology Symposium: Uterine infection: Linking infection and innate immunity with infertility in the high-producing dairy cow. J. Anim. Sci. 93:2021-2033.

Caporaso, J. G., C. L. Lauber, W. A. Walters, D. Berg-Lyons, J. Huntley, N. Fierer, S. M. Owens, J. Betley, L. Fraser, M. Bauer, N. Gormley, J. A. Gilbert, G. Smith, and R. Knight. 2012. Ultrahigh-throughput microbial community analysis on the Illumina HiSeq and MiSeq platforms. ISME J. 6:1621-1624.

Cole, J. R., Q. Wang, E. Cardenas, J. Fish, B. Chai, R. J. Farris, A. S. Kulam-Syed-Mohideen, D. M. McGarrell, T. Marsh, G. M. Garrity, and J. M. Tiedje. 2009. The ribosomal database project: Improved alignments and new tools for rRNA analysis. Nucleic Acids Res. 37:D141-D145.

De Rycke, J., and E. Oswald. 2001. Cytolethal distending toxin (CDT): A bacterial weapon to control host cell proliferation? FEMS Microbiol. Lett. 203:141-148.

DeSantis, T. Z., P. Hugenholtz, N. Larsen, M. Rojas, E. L. Brodie, K. Keller, T. Huber, D. Dalevi, P. Hu, and G. L. Andersen. 2006. Greengenes, a chimera-checked 16S rRNA gene database and workbench compatible with ARB. Appl. Environ. Microbiol. 72:5069-5072.

Deszo, E. L., S. M. Steenbergen, D. I. Freedberg, and E. R. Vimr. 2005. Escherichia coli $\mathrm{K} 1$ polysialic acid O-acetyltransferase gene, neuO, and the mechanism of capsule form variation involving a mobile contingency locus. Proc. Natl. Acad. Sci. USA 102:5564-5569.

Dubuc, J., T. F. Duffield, K. E. Leslie, J. S. Walton, and S. J. LeBlanc. 2010. Definitions and diagnosis of postpartum endometritis in dairy cows. J. Dairy Sci. 93:5225-5233.

Fricke, P. M., D. Z. Caraviello, K. A. Weigel, and M. L. Welle. 2003. Fertility of dairy cows after resynchronization of ovulation at three intervals following first timed insemination. J. Dairy Sci. 86:39413950. 
Gilbert, R. O., S. T. Shin, C. L. Guard, H. N. Erb, and M. Frajblat. 2005. Prevalence of endometritis and its effects on reproductive performance of dairy cows. Theriogenology 64:1879-1888.

Gunn, J. S., K. B. Lim, J. Krueger, K. Kim, L. Guo, M. Hackett, and S. I. Miller. 1998. PmrA-PmrB-regulated genes necessary for 4-aminoarabinose lipid A modification and polymyxin resistance. Mol. Microbiol. 27:1171-1182.

Helander, I. M., K. Nummila, I. Kilpelainen, and M. Vaara. 1995. Increased substitution of phosphate groups in lipopolysaccharides and lipid A of polymyxin-resistant mutants of Salmonella typhimurium and Escherichia coli. Prog. Clin. Biol. Res. 392:15-23.

Herschman, H. R., and D. R. Helinski. 1967. 'Purification and characterization of colicin E2 and colicin E3'. J. Biol. Chem. 242:53605368.

Hunter, S. J., S. Easton, V. Booth, B. Henderson, W. G. Wade, and J. M. Ward. 2011. Selective removal of human DNA from metagenomic DNA samples extracted from dental plaque. J. Basic Microbiol. 51:442-446.

Jeon, S. J., A. Vieira-Neto, M. Gobikrushanth, R. Daetz, R. D. Mingoti, A. C. Parize, S. L. de Freitas, A. N. da Costa, R. C. Bicalho, S. Lima, K. C. Jeong, and K. N. Galvao. 2015. Uterine microbiota progression from calving until establishment of metritis in dairy cows. Appl. Environ. Microbiol. 81:6324-6332.

Kasimanickam, R., T. F. Duffield, R. A. Foster, C. J. Gartley, K. E. Leslie, J. S. Walton, and W. H. Johnson. 2004. Endometrial cytology and ultrasonography for the detection of subclinical endometritis in postpartum dairy cows. Theriogenology 62:9-23.

Kent, W. J. 2002. BLAT-The BLAST-like alignment tool. Genome Res. 12:656-664.

Knudsen, L. R., C. C. Karstrup, H. G. Pedersen, J. S. Agerholm, T. K. Jensen, and K. Klitgaard. 2015. Revisiting bovine pyometra-new insights into the disease using a culture-independent deep sequencing approach. Vet. Microbiol. 175:319-324.

LeBlanc, S. J., K. D. Lissemore, D. F. Kelton, T. F. Duffield, and K. E. Leslie. 2006. Major advances in disease prevention in dairy cattle. J. Dairy Sci. 89:1267-1279.

Machado, V. S., G. Oikonomou, M. L. Bicalho, W. A. Knauer, R. Gilbert, and R. C. Bicalho. 2012. 'Investigation of postpartum dairy cows' uterine microbial diversity using metagenomic pyrosequencing of the 16S rRNA gene'. Vet. Microbiol. 159:460-469.

McDougall, S., R. Macaulay, and C. Compton. 2007. Association between endometritis diagnosis using a novel intravaginal device and reproductive performance in dairy cattle. Anim. Reprod. Sci. 99:9-23.

Moreira, F., C. Orlandi, C. A. Risco, R. Mattos, F. Lopes, and W. W. Thatcher. 2001. Effects of presynchronization and bovine somatotropin on pregnancy rates to a timed artificial insemination protocol in lactating dairy cows. J. Dairy Sci. 84:1646-1659.

Nadkarni, M. A., F. E. Martin, N. A. Jacques, and N. Hunter. 2002 Determination of bacterial load by real-time PCR using a broadrange (universal) probe and primers set. Microbiology 148:257-266.

Nummila, K., I. Kilpelainen, U. Zahringer, M. Vaara, and I. M. Helander. 1995. Lipopolysaccharides of polymyxin B-resistant mutants of Escherichia coli are extensively substituted by 2-aminoethyl pyrophosphate and contain aminoarabinose in lipid A. Mol. Microbiol. 16:271-278.

Overbeek, R., R. Olson, G. D. Pusch, G. J. Olsen, J. J. Davis, T. Disz, R. A. Edwards, S. Gerdes, B. Parrello, M. Shukla, V. Vonstein, A. R. Wattam, F. Xia, and R. Stevens. 2014. The SEED and the Rapid Annotation of microbial genomes using Subsystems Technology (RAST). Nucleic Acids Res. 42:D206-D214.

Parks, D. H., G. W. Tyson, P. Hugenholtz, and R. G. Beiko. 2014. STAMP: Statistical analysis of taxonomic and functional profiles. Bioinformatics 30:3123-3124.

Peng, Y., Y. Wang, S. Hang, and W. Zhu. 2013. Microbial diversity in uterus of healthy and metritic postpartum Holstein dairy cows. Folia Microbiol. (Praha). 58:593-600. https://doi. org/10.1007s12223-013-0238-6.

Potter, T. J., J. Guitian, J. Fishwick, P. J. Gordon, and I. M. Sheldon. 2010. Risk factors for clinical endometritis in postpartum dairy cattle. Theriogenology 74:127-134.
Pruesse, E., C. Quast, K. Knittel, B. M. Fuchs, W. Ludwig, J. Peplies, and F. O. Glöckner. 2007. SILVA: A comprehensive online resource for quality checked and aligned ribosomal RNA sequence data compatible with ARB. Nucleic Acids Res. 35:7188-7196.

Pursley, J. R., M. O. Mee, and M. C. Wiltbank. 1995. Synchronization of ovulation in dairy cows using PGF2alpha and GnRH. Theriogenology 44:915-923.

Raetz, C. R., C. M. Reynolds, M. S. Trent, and R. E. Bishop. 2007 Lipid A modification systems in gram-negative bacteria. Annu. Rev. Biochem. 76:295-329.

Santos, T. M., and R. C. Bicalho. 2012. Diversity and succession of bacterial communities in the uterine fluid of postpartum metritic, endometritic and healthy dairy cows. PLoS One 7:e53048.

Santos, T. M., R. O. Gilbert, and R. C. Bicalho. 2011. Metagenomic analysis of the uterine bacterial microbiota in healthy and metritic postpartum dairy cows. J. Dairy Sci. 94:291-302.

Sens, A., and W. Heuwieser. 2013. Presence of Escherichia coli, Trueperella pyogenes, alpha-hemolytic streptococci, and coagulase-negative staphylococci and prevalence of subclinical endometritis. J. Dairy Sci. 96:6347-6354.

Sheldon, I. M., G. S. Lewis, S. LeBlanc, and R. O. Gilbert. 2006 Defining postpartum uterine disease in cattle. Theriogenology 65:1516-1530

Sheldon, I. M., and M. H. Roberts. 2010. Toll-like receptor 4 mediates the response of epithelial and stromal cells to lipopolysaccharide in the endometrium. PLoS One 5:e12906.

Sheldon, I. M., A. N. Rycroft, B. Dogan, M. Craven, J. J. Bromfield, A. Chandler, M. H. Roberts, S. B. Price, R. O. Gilbert, and K. W. Simpson. 2010. Specific strains of Escherichia coli are pathogenic for the endometrium of cattle and cause pelvic inflammatory disease in cattle and mice. PLoS One 5:e9192.

Tadepalli, S., S. K. Narayanan, G. C. Stewart, M. M. Chengappa and T. G. Nagaraja. 2009. Fusobacterium necrophorum: A ruminal bacterium that invades liver to cause abscesses in cattle. Anaerobe 15:36-43.

Tóth, I., F. Herault, L. Beutin, and E. Oswald. 2003. Production of cytolethal distending toxins by pathogenic Escherichia coli strains isolated from human and animal sources: Establishment of the existence of a new CDT variant (Type IV). J. Clin. Microbiol. 41:4285-4291.

Vimr, E., and C. Lichtensteiger. 2002. To sialylate, or not to sialylate: That is the question. Trends Microbiol. 10:254-257.

Wagener, K., T. Grunert, I. Prunner, M. Ehling-Schulz, and M. Drillich. 2014. Dynamics of uterine infections with Escherichia coli, Streptococcus uberis and Trueperella pyogenes in post-partum dairy cows and their association with clinical endometritis. Vet. J. 202:527-532.

Welch, R. A., V. Burland, G. Plunkett 3rd, P. Redford, P. Roesch, D. Rasko, E. L. Buckles, S. R. Liou, A. Boutin, J. Hackett, D Stroud, G. F. Mayhew, D. J. Rose, S. Zhou, D. C. Schwartz, N. T. Perna, H. L. Mobley, M. S. Donnenberg, and F. R. Blattner. 2002. Extensive mosaic structure revealed by the complete genome sequence of uropathogenic Escherichia coli. Proc. Natl. Acad. Sci. USA 99:17020-17024.

Westermann, S., M. Drillicha, T. B. Kaufmann, L. V. Madoz, and W. Heuwieser. 2010. A clinical approach to determine false positive findings of clinical endometritis by vaginoscopy by the use of uterine bacteriology and cytology in dairy cows. Theriogenology $74: 1248-1255$.

Wilke, A., T. Harrison, J. Wilkening, D. Field, E. M. Glass, N. Kyrpides, K. Mavrommatis, and F. Meyer. 2012. The M5nr: A novel non-redundant database containing protein sequences and annotations from multiple sources and associated tools. BMC Bioinformatics 13:141.

Williams, E. J., D. P. Fischer, D. U. Pfeiffer, G. C. England, D. E. Noakes, H. Dobson, and I. M. Sheldon. 2005. Clinical evaluation of postpartum vaginal mucus reflects uterine bacterial infection and the immune response in cattle. Theriogenology 63:102-117. 